\title{
Impact of nuclear "pasta" on neutrino transport in collapsing stellar cores
}

\author{
Hidetaka Sonoda ${ }^{a, b}$, Gentaro Watanabe ${ }^{c, b}$, Katsuhiko Sato ${ }^{a, d}$, \\ Tomoya Takiwaki ${ }^{a}$, Kenji Yasuoka ${ }^{e}$, and Toshikazu Ebisuzaki ${ }^{b}$ \\ ${ }^{a}$ Department of Physics, University of Tokyo, Tokyo 113-0033, Japan \\ ${ }^{b}$ The Institute of Chemical and Physical Research (RIKEN), Saitama 351-0198, Japan \\ ${ }^{c}$ NORDITA, Blegdamsvej 17, DK-2100 Copenhagen Ø, Denmark \\ ${ }^{d}$ Research Center for the Early Universe, University of Tokyo, Tokyo 113-0033, Japan \\ ${ }^{e}$ Department of Mechanical Engineering, Keio University, Yokohama, 223-8522, Japan
}

(Dated: October 24, 2018)

\begin{abstract}
Nuclear "pasta", nonspherical nuclei in dense matter, is predicted to occur in collapsing supernova cores. We show how pasta phases affect the neutrino transport cross section via weak neutral current using several nuclear models. This is the first calculation of the neutrino opacity of the phases with rod-like and slab-like nuclei taking account of finite temperature effects, which are well described by the quantum molecular dynamics. We also show that pasta phases can occupy 10-20\% of the mass of supernova cores in the later stage of the collapse.
\end{abstract}

PACS numbers: 26.50.+x, 25.30.Pt, 97.60.Bw, 13.15.+g

Interactions between neutrinos and matter are one of the key elements for understanding the mechanism of core-collapse supernovae. In the collapse phase, neutrinomatter interactions play a crucial role for explosion: the efficiency of the trapping of neutrinos inside the collapsing core governs its lepton fraction $Y_{L}$ (the ratio of the number density of leptons to that of baryons) and hence the mass of the inner core at bounce. The latter quantity determines the initial position and the strength of the shock wave [1].

In the neutrino-trapping stage, interactions between neutrinos and matter are dominated by coherent scattering of neutrinos from heavy nuclei via the weak neutral current, which we focus on in this article. In the case of scattering of neutrinos by an isolated nucleus, neutrinos are coherently scattered by nucleons in the nucleus provided the wavelength of neutrinos is much larger than the radius of the nucleus $r_{N}$. Therefore, roughly speaking, the cross section per nucleon is enhanced by a factor of $A[2,[3]$, where $A$ is the mass number of the nucleus, due to the fact that the scattering is coherent. However, when neutrinos are scattered by matter in the core, not by an isolated nucleus, more than one nucleus would contribute to the scattering amplitude and the coherence can be disturbed if the internuclear distance is comparable to or smaller than the wavelength of neutrinos [4]. This correction is due to the spatial correlation among nuclei. Especially, at higher densities, nuclei form a lattice because of the strong Coulomb forces among nuclei and thus the correlation among them is very different from that of the liquid phase at lower densities.

More interestingly, the nuclear shape itself would change from sphere to rod or slab, etc., at subnuclear den- sities in the collapsing supernova core [5, $[6,7,6,8,9,10]$. These nonspherical nuclei are referred to as "nuclear pasta". Transitions to pasta phases also change the correlation among nuclei and hence the neutrino opacity.

At the present time, the effects of nuclear pasta on the neutrino opacity have yet to be understood completely. One of the important questions to be clarified is how the neutrino opacity for the conventional models of dense matter used in supernova simulations is modified by taking account of the pasta phases. Horowitz et al. have studied a related problem using the framework of quantum molecular dynamics (QMD) [1] , but they have not reproduced the phases with rod-like and slab-like nuclei and those with rod-like and spherical bubbles probably due to the use of a short screening length (10 fm) compared to the internuclear distance (see a discussion in Ref. [12]). Therefore the above question is still open.

We calculate the cross section for elastic neutrino scattering from pasta phases via weak neutral current [13]. After averaging over the spin states of nucleons, resulting expression of the neutrino cross section per nucleon to the first order is

$$
\frac{1}{N} \frac{d \sigma}{d \Omega}(\mathbf{q})=\frac{G_{F}^{2} E_{\nu}^{2}}{4 \pi^{2}}(1+\cos \theta) c_{v}^{(n)^{2}} \bar{S}(\mathbf{q}),
$$

where $N$ is the number of nucleons in the system, $G_{F}$ is the Fermi coupling constant, $E_{\nu}$ is the energy of neutrinos, $\theta$ is the scattering angle, $c_{v}^{(n)}\left(c_{v}^{(p)}\right)$ is the vector coupling constant of neutrons (protons) to the weak neutral current $\left(c_{v}^{(n)}=-1 / 2, c_{v}^{(p)}=1 / 2-2 \sin ^{2} \theta_{W}, \sin ^{2} \theta_{W}=\right.$ $0.23), \hbar \mathbf{q}$ is the momentum transfer, and $\bar{S}(\mathbf{q})$ is defined as follows:

$$
\bar{S}(\mathbf{q})=\frac{1}{c_{v}^{(n)^{2}}}\left\{c_{v}^{(n)^{2}}\left(x_{n}^{2} S_{n n}(\mathbf{q})+x_{p} x_{n}\right)+c_{v}^{(p)^{2}}\left(x_{p}^{2} S_{p p}(\mathbf{q})+x_{p} x_{n}\right)+c_{v}^{(n)} c_{v}^{(p)} x_{p} x_{n}\left(S_{p n}(\mathbf{q})+S_{n p}(\mathbf{q})-2\right)\right\}
$$


In Eq. (2), $x_{p}\left(x_{n}\right)$ is the proton (neutron) fraction of the system, $S_{A B}(\mathbf{q})$ with $A, B=n$ or $p$ is the partial static structure factor of nucleons of species $A$ and $B$,

$$
S_{A B}(\mathbf{q})=1+\rho \int d^{3} r e^{i \mathbf{q} \cdot \mathbf{r}}\left\{g_{A B}(\mathbf{r})-1\right\},
$$

where $\rho$ is the average number density of nucleons in the system and $g_{A B}$ is the two-body distribution function of species $A$ and $B$ defined as follows,

$$
\begin{aligned}
& g_{A B}(\mathbf{r}) \\
\equiv & \frac{1}{\rho_{A} \rho_{B}} \frac{1}{V} \int d^{3} r^{\prime}\left\langle\psi_{A}^{\dagger}\left(\mathbf{r}+\mathbf{r}^{\prime}\right) \psi_{B}^{\dagger}(\mathbf{r}) \psi_{B}(\mathbf{r}) \psi_{A}\left(\mathbf{r}+\mathbf{r}^{\prime}\right)\right\rangle .
\end{aligned}
$$

In Eq. (4), $\langle\cdots\rangle$ denotes the statistical average, $\psi_{A}\left(\psi_{B}\right)$ is the field operator of particle $A(B), \rho_{A}\left(\rho_{B}\right)$ is number density of $A(B)$, and $V$ is the volume of the system. If we ignore the contribution of the axial vector current, the cross section for the scattering of neutrinos by a single neutron is given by

$$
\frac{d \sigma_{n}}{d \Omega}=\frac{G_{F}^{2} E_{\nu}^{2}}{4 \pi^{2}} c_{v}^{(n)^{2}}(1+\cos \theta) .
$$

Comparing Eqs. (11) and (5), we see that $\bar{S}(\mathbf{q})$ is the amplification factor of the cross section per nucleon due to the structure of nuclear matter. We normalize Eq. (2) by $c_{v}^{(n)}$ because neutrons dominantly contribute to the vector current.

In the field of simulation of supernovae, researchers often use transport cross section defined as

$$
\frac{d \sigma_{t}}{d \Omega}(\mathbf{q}) \equiv \frac{d \sigma}{d \Omega}(1-\cos \theta),
$$

which means the efficiency of the momentum transport from neutrinos to matter. Note that the cross section depends on the relative direction between the momentum of neutrinos and the symmetry axis of the pasta phases as shown later in Eq. (10). However, collapsing cores are polycrystalline; the symmetry axes of grains are randomly oriented and the size of the grains would be much smaller than the mean free path of neutrinos. Thus the neutrino opacity of supernova cores would be well characterized by the angle-average of $\bar{S}(\mathbf{q})$ (an average over the scattering angle $\theta$ ),

$$
\left\langle\bar{S}\left(E_{\nu}\right)\right\rangle \equiv \frac{3}{32 \pi^{2}} \int d \Omega_{i} d \Omega_{f}\left(1-\cos ^{2} \theta\right) \bar{S}(\mathbf{q})
$$

where $\Omega_{i}$ and $\Omega_{f}$ are integrated over initial and final directions of neutrinos. The above quantity is related to the transport cross section $\sigma_{t}$ per nucleon as

$$
\sigma_{t}=\left\langle\bar{S}\left(E_{\nu}\right)\right\rangle \sigma_{t}^{0},
$$

where $\sigma_{t}^{0}$ is the total transport cross section of one neutron via the vector current,

$$
\sigma_{t}^{0}=\frac{2 G_{F}^{2} E_{\nu}^{2}}{3 \pi} c_{v}^{(n)^{2}}
$$

Thus $\left\langle\bar{S}\left(E_{\nu}\right)\right\rangle$ is an amplification factor of the total transport cross section.

When nuclei form a crystalline lattice, the partial static structure factor $S_{A B}(\mathbf{q})$, within the Wigner-Seitz (WS) approximation, can be written as

$$
\begin{aligned}
S_{A B}(\mathbf{q})=1 & -\frac{\rho}{\rho_{A}} \delta_{A B} \\
& +\frac{1}{x_{A} x_{B}} F_{A}(-\mathbf{q}) F_{B}(\mathbf{q}) \frac{(2 \pi)^{3}}{\rho V_{\mathrm{WS}}^{2}} \sum_{\mathbf{G}} \delta^{(3)}(\mathbf{q}-\mathbf{G}),
\end{aligned}
$$

where $\mathbf{G}$ is the reciprocal lattice vector of each structure, $x_{A}\left(x_{B}\right)$ is the fraction of particles of species $A(B)$ in the system, $V_{\mathrm{WS}}$ is the volume of the WS cell, $F_{A}\left(F_{B}\right)$ is the form factor of a single nucleus for species $A(B)$ defined as

$$
F_{A}(\mathbf{q}) \equiv \int_{V_{\mathrm{n}}} d^{3} r \rho_{A}^{(N)}(\mathbf{r}) \cdot e^{i \mathbf{q} \cdot \mathbf{r}}
$$

Here $\rho_{A}^{(N)}$ is the difference of densities of species $A$ inside and outside nuclei, and the integration $\int_{V_{\mathrm{n}}}$ is over the volume $V_{\mathrm{n}}$ of one nucleus. In the following, we use the above expressions to calculate $\left\langle\bar{S}\left(E_{\nu}\right)\right\rangle$ for models given by ourselves [14, 15] and by Lattimer and Swesty [16] in which lattice structure is treated within the WS approximation. In the present calculation, however, we take account of the lattice structure explicitly: we assume a BCC lattice of spherical nuclei and bubbles, a triangular lattice of rod-like nuclei and bubbles, and a one-dimensional lattice of slab-like nuclei. These assumptions are justified by the fact that the Coulomb energy for the assumed lattice structure is well reproduced by the WS approximation [17]. In the following numerical calculations, the summation about $\mathbf{G}$ in Eq. (10) is done for the six smallest reciprocal lattice vectors because the contribution of higher orders is negligible in the present situation, where the nuclear volume fraction is large (the contributions of the fifth and sixth orders are already $10^{-3}$ smaller than that of the first order).

To see the effects of existence of nuclear pasta clearly, we compare $\left\langle\bar{S}\left(E_{\nu}\right)\right\rangle$ for two cases: (1) we take account of the pasta phases, i.e., the phases with rod-like nuclei, slab-like nuclei, and rod-like bubbles, in addition to the phases with spherical nuclei and bubbles; (2) we only take account of the phases with spherical nuclei and bubbles (here referred to as the no pasta case). For case (1), we use nuclear data of supernova matter at zero temperature and at $Y_{L}=0.3$ obtained in Refs. [14 and 15]. In these references we used a liquid drop model, which is based on that developed by Baym, Bethe, and Pethick (BBP) [18. Here we use results for standard values of parameters for the surface tension and the chemical potential in this model. Case (2) is newly calculated for the same parameters using the same model and taking account of only spherical nuclei and bubbles. In Fig. 1 we show the results of these two cases. We can see the common tendencies that $\left\langle\bar{S}\left(E_{\nu}\right)\right\rangle$ is zero below $E_{\nu} \simeq 25 \mathrm{MeV}$, rapidly increases at $25-30 \mathrm{MeV}$, has a peak around $30-40 \mathrm{MeV}$, 


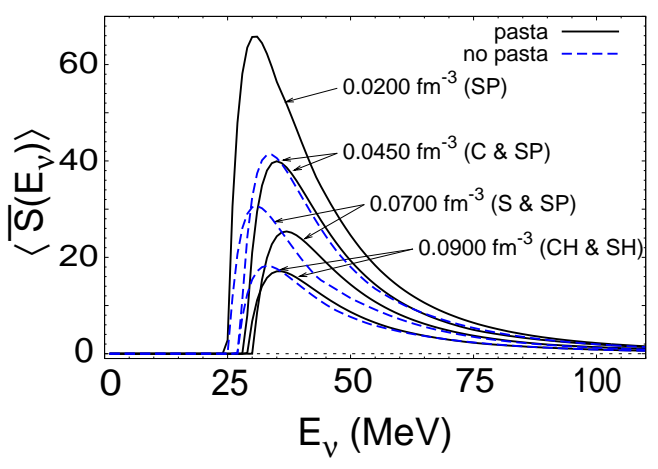

FIG. 1: (Color online) $\left\langle\bar{S}\left(E_{\nu}\right)\right\rangle$ at $Y_{L}=0.3$ calculated for a liquid drop model based on BBP with a typical parameter set given by Ref. [14]. The abbreviations SP, C, S, CH, and SH stand for phases with spherical nuclei, cylindrical nuclei, slablike nuclei, cylindrical holes and spherical holes, respectively.

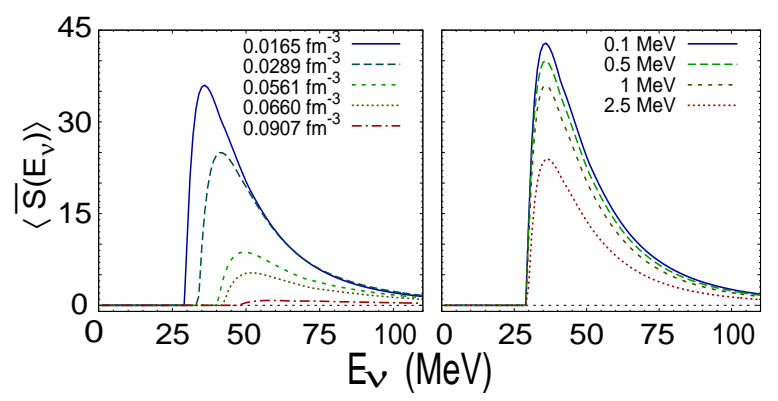

FIG. 2: (Color online) $\left\langle\bar{S}\left(E_{\nu}\right)\right\rangle$ at $x_{p}=0.3$ calculated for EOS by Lattimer and Swesty (LS-EOS) [16]. In the left panel, the temperature is fixed at $1 \mathrm{MeV}$. In the right panel, the number density of nucleons is fixed at $0.0165 \mathrm{fm}^{-3}$.

falls relatively gently, and approaches $x_{n}+\left(c_{v}^{(p)} / c_{v}^{(n)}\right)^{2} x_{p}$ above $100 \mathrm{MeV}$ [19]. Peak height monotonically decreases with increasing density. This is simply because the nucleon density distribution becomes more uniform. Striking differences between the results for the cases with and without the pasta phases are as follows. The peak height decreases and the peak energy increases when one takes account of the pasta phases. The former is caused by the disappearance of the coherence along the axis of cylindrical nuclei and in the plane of slab-like nuclei. The latter is due to smaller values of the lattice constant for the phases with rod-like and slab-like nuclei compared to those for the phases with spherical nuclei and bubbles; this is a general property observed in a number of previous calculations [14, 15, 17, 20, 21].

In Fig. 2 we show $\left\langle\bar{S}\left(E_{\nu}\right)\right\rangle$ for the equation of state (EOS) by Lattimer and Swesty (LS-EOS) [16] at a proton fraction $x_{p}=0.3$ to see effects of finite temperatures within the liquid drop approach [22]. The left panel shows the density dependence and the right one the temperature dependence. We note that, in this calculation, nuclei are assumed to be spherical and the other struc- tures including the spherical bubbles are not taken into account. In the present calculation, when we take an average of $S_{A B}(\mathbf{q})$ over the scattering angle, we multiply $S_{A B}(\mathbf{q})$ by the Debye-Waller factor given by Ref. 23] and use the relation $q^{2}=2 E_{\nu}^{2}(1-\cos \theta)$. The right panel shows that $\left\langle\bar{S}\left(E_{\nu}\right)\right\rangle$ falls monotonically with increasing temperature. This is due to thermal fluctuations of the position of nuclei from equilibrium lattice points described by the Debye-Waller factor and the smoothed nucleon density profile at higher temperatures. Although these two kinds of finite temperature effects can be (even partially) incorporated within the liquid drop model such as LS-EOS, there is another kind of finite temperature effect that is hard to be described by the liquid drop model: disorder. Figure 2 exhibits a steep decline of the peak on the low energy side. Disorder will broaden the peak of $\left\langle\bar{S}\left(E_{\nu}\right)\right\rangle$ and will produce a spectral tail on the low energy side since the disorder of lattice contributes to $\left\langle\bar{S}\left(E_{\nu}\right)\right\rangle$ at wavelengths necessarily longer than the lattice constant. The QMD can describe the finite temperature effects including disorder beyond the limit of liquid drop models. Next, we show $\left\langle\bar{S}\left(E_{\nu}\right)\right\rangle$ calculated for QMD simulations as more realistic results.

Here we briefly summarize QMD models and computation techniques. We use nuclear forces given by Maruyama et al. 24] (model 1) and by Chikazumi et al. [25] and Kido et al. [26] (model 2) with medium EOS parameter sets 27]. Model 1 is used in our previous works [9, 10, 28, 29]. Model 2 is newly adopted for studying the dependence of the phase diagram on nuclear forces within the framework of QMD [30]. Parameter sets for both the models are determined by fitting the saturation properties of nuclear matter, the properties of finite nuclei in the ground state, and the energy dependence of the optical potential. In the Hamiltonian of model 2, there is an extra term depending on the density gradient, which is not included in that of model 1 . Procedures to obtain the equilibrium states of hot nuclear matter by QMD are explained in Ref. [9] for model 1 and in Ref. [30] for model 2.

We calculate the angle-averaged radial distribution function $g_{A B}(r)$ for snapshots of QMD simulations with 16384 (or 2048) nucleons at a proton fraction $x_{p}=0.3$ [31]. In calculating $g_{A B}(r)$, we place $5^{3}$ (or $10^{3}$ ) resulting simulation boxes, each containing 16384 (or 2048) nucleons, to enlarge the cutoff radius of $g_{A B}(r)$; we take the cutoff radius to be greater than $100 \mathrm{fm}$. Then we obtain $S_{A B}(q)$ by calculating the Fourier transform of $g_{A B}(r)$ multiplied by a Hanning window [32], which removes ripples caused by Fourier transforming $g_{A B}(r)$ with a discrete change at the finite cut-off radius.

In Fig. 3 we show $\left\langle\bar{S}\left(E_{\nu}\right)\right\rangle$ at a fixed temperature $(T=1 \mathrm{MeV})$ for QMD model 1 (left panel) and at a fixed density $\left(0.0660 \mathrm{fm}^{-3}\right)$ for QMD model 2 (right panel), respectively. From these figures, we observe two important characteristics of $\left\langle\bar{S}\left(E_{\nu}\right)\right\rangle$ calculated for the results of the QMD simulations. One is that the peak is lower and broader than the results for the liquid drop models 


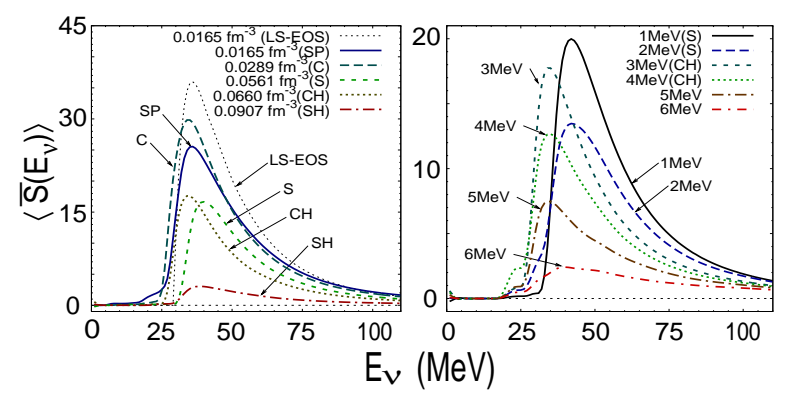

FIG. 3: (Color online) $\left\langle\bar{S}\left(E_{\nu}\right)\right\rangle$ at $x_{p}=0.3$ calculated for QMD model 1 (left panel) and model 2 (right panel). Abbreviations are the same as in Fig. 1. The left panel shows $\left\langle\bar{S}\left(E_{\nu}\right)\right\rangle$ for each pasta phase at $T=1 \mathrm{MeV}$. For comparison we also show $\left\langle\bar{S}\left(E_{\nu}\right)\right\rangle$ at $0.0165 \mathrm{fm}^{-3}$ and $T=1 \mathrm{MeV}$ for LSEOS. The right panel shows $\left\langle\bar{S}\left(E_{\nu}\right)\right\rangle$ for various temperatures at a fixed density $0.0660 \mathrm{fm}^{-3}$.

shown before (see the lines for SP and LS-EOS in the left panel of Fig. (3). This is due to lattice disorder and irregularities of nuclei, which can be incorporated by QMD, but not by the liquid drop models. The former (latter) enhances the cross section of low (high) energy neutrinos, while they reduce that of neutrinos around peak energy. It is noticeable that, due to the former effect, $\left\langle\bar{S}\left(E_{\nu}\right)\right\rangle$ calculated for the QMD results has nonzero values in the low energy region of $E_{\nu} \lesssim 25 \mathrm{MeV}$ in some cases while $\left\langle\bar{S}\left(E_{\nu}\right)\right\rangle$ shown in Figs. 1 and 2 vanishes in this region. The other important characteristic is that, as shown in the left panel of Fig. 3, the peak height does not necessarily decrease with increasing density unlike the results for the liquid drop models shown in Figs. 1 1 and2, This nonmonotonic behavior is observed between the phases with spherical nuclei and rod-like ones, and also between the phases with slab-like nuclei and rod-like bubbles. Let us first consider the latter case. In the results of the QMD simulations, slab-like nuclei form a layered lattice and rod-like bubbles form a triangular lattice. The ratio of the number of the smallest reciprocal lattice vectors of a layered lattice to that of a triangular one is 1:3. This larger number of the lowest order of the reciprocal lattice vectors for the phase with rod-like bubbles might explain the larger peak height compared to the phase with slablike nuclei [31]. For the same reason, it is possible that $\left\langle\bar{S}\left(E_{\nu}\right)\right\rangle$ increases with a transition from the phase with slab-like nuclei to that with rod-like bubbles caused by an increase of temperature as suggested in the right panel of Fig. 3. Here nuclei adopt a slab-like shape at 1-2 MeV and nuclear structure transforms to rod-like bubbles at 3-4 MeV, above which nuclei deform and nuclear surface cannot be identified. On the other hand, the reversal of the peak height between the phases with spherical nuclei and rod-like ones would be explained by the position of spherical nuclei in the former being easy to fluctuate compared to that of rod-like nuclei in the latter, where the nuclear matter region is connected in one direction and this acts to suppress the fluctuation in the transverse
TABLE I: The mass of the region where pasta phases would appear $\left(M_{\text {pasta }}\right)$ and that where nuclei exist $\left(M_{\text {nuc }}\right)$ just before the bounce in the typical supernova simulations for $T /|W|=0$ and 0.01. Radius means the distance of possible regions for pasta phases from the center of the core. Mass is measured by the solar mass $M_{\odot}$. Ratio is defined as $M_{\text {pasta }} / M_{\text {nuc }}$.

\begin{tabular}{c|cccc}
$T /|W|$ & Radius $(\mathrm{cm})$ & $M_{\text {pasta }}\left(M_{\odot}\right)$ & $M_{\text {nuc }}\left(M_{\odot}\right)$ & Ratio \\
\hline 0 & $1.1-1.3 \times 10^{6}$ & 0.13 & 0.92 & 0.14 \\
0.01 & $0.8-1.6 \times 10^{6}$ & 0.30 & 1.29 & 0.23 \\
\hline
\end{tabular}

\section{direction.}

Finally we estimate the possible mass of the pasta phases in supernova cores to examine their importance on the core-collapse supernova explosions. We have performed two-dimensional axisymmetric simulations of rotational core collapse supernovae. The initial condition is taken from the profile of the $15 M_{\odot}$ nonrotating progenitor with solar metalicity at zero-age main sequence (ZAMS) by Woosley et al. 33]. The mass of the core is $1.58 M_{\odot}$. Rotation is then added in the same way as in Ref. 34]. Here we set $T /|W|=0$ and 0.01 , where $T /|W|$ is a ratio of the rotational kinetic energy $T$ to the gravitaional binding energy $W$ of the core. These values of $T /|W|$ are typical and the latter one is also suggested by simulations of the evolution of massive stars with a typical rotational velocity on the equator at ZAMS, $\sim 200 \mathrm{~km} / \mathrm{s}[33,35]$. The magnetic field is set to be zero. We use an EOS by Shen et al. [36]. Other basic equations and input physics are shown in Refs. 34] and 37].

In Table【, we show the mass $M_{\text {pasta }}$ of the pasta phases just before the bounce. The region where the matter consists of the pasta phases is defined by the following two conditions: 1) nuclei exist and 2) the volume fraction of nuclei is larger than $1 / 8$, which is a condition for the fission instability of spherical nuclei giving a reasonable estimate of the density at which matter starts to consist of nonspherical nuclei [38]. Note that the EOS used in our simulations only incorporates the phases with spherical nuclei and uniform nuclear matter and it does not take account of the phase with spherical bubbles and any other pasta phases. Therefore values of $M_{\text {pasta }}$ in Table \are lower limits in our simulations: some region of uniform nuclear matter close to a boundary with the phase with nuclei in our simulations corresponds to the pasta phases. We also show the mass $M_{\text {nuc }}$ of the region where nuclei exist in the core because the pasta phases modify the cross section of elastic neutrino scattering, which is dominated by the contribution of nuclei. It is notable that about $10-20 \%$ of spherical nuclei are replaced by the pasta nuclei in both the rotating and no-rotating cases. In addition, the moderate rotation in the core extend the region of pasta phases by centrifugal force [39].

In summary we have shown how pasta phases affect energy-dependent cross sections for coherent scattering of neutrinos in collapsing cores. At zero temperature, existence of the pasta phases instead of the phases with spherical neuclei and bubbles increases the energy of the 
peak of the cross section at $E_{\nu} \simeq 30-40 \mathrm{MeV}$ and decreases the peak height. They lead to a reduction in the opacity of the core for low energy neutrinos of $E_{\nu} \lesssim 30$ $\mathrm{MeV}$. At non-zero temperatures, the peak height further decreases but increases the cross section of neutrinos at energy lower and higher than that of the peak. The pasta phases, which amount to 10-20\% of the mass of supernova cores, would modify the lepton fraction of the core. The next step is to construct a systematic numerical table or a useful fitting formula of the cross section for elastic neutrino scattering in the pasta phases and perform hydrodynamic core collapse simulations, which incorporate the effects of the pasta phases on the neutrino cross section studied in the present article.

We are grateful to T. Maruyama, S. Chikazumi, K. Iida, and K. Oyamatsu for helpful discussions. We also thanks Chris Pethick for valuable comments. This work was supported in part by JSPS, by the Junior Research Associate Program in RIKEN through Research Grant No. J130026, by Grants-in-Aid for Scientific Research provided by the Ministry of Education, Culture, Sports, Science and Technology through Research Grants No. 14-7939 and No. 14079202, and by the Nishina Memorial Foundation. Parts of the simulations were performed by the RIKEN Super Combined Cluster System.
[1] H. A. Bethe, Rev. Mod. Phys. 62, 801 (1990).

[2] D. Z. Freedman, Phys. Rev. D 9, 1389 (1974).

[3] K. Sato, Prog. Theor. Phys. 54, 1325 (1975).

[4] N. Itoh, Prog. Theor. Phys. 54, 1580 (1975).

[5] D. G. Ravenhall, C. J. Pethick, and J. R. Wilson, Phys. Rev. Lett. 50, 2066 (1983).

[6] M. Hashimoto, H. Seki, and M. Yamada, Prog. Theor. Phys. 71, 320 (1984).

[7] R. D. Williams and S. E. Koonin, Nucl. Phys. A435, 844 (1985).

[8] M. Lassaut, H. Flocard, P. Bonche, P. H. Heenen, and E. Suraud, Astron. Astrophys. 183, L3 (1987).

[9] G. Watanabe, K. Sato, K. Yasuoka, and T. Ebisuzaki, Phys. Rev. C 69, 055805 (2004).

[10] G. Watanabe, T. Maruyama, K. Sato, K. Yasuoka, and T. Ebisuzaki, Phys. Rev. Lett. 94, 031101 (2005).

[11] C. J. Horowitz, M. A. Perez-Garcia, J. Carriere, D. K. Berry, and J. Piekarewicz, Phys. Rev. C 70, 065806 (2004).

[12] G. Watanabe, to appear in the Proceedings of the Tours symposium on nuclear physics VI (TOURS 2006), edited by H. Utsunomiya (nucl-th/0610116).

[13] Here we are interested in coherent scattering of neutrinos from heavy nuclei at subnuclear densities and thus a typical value of the momentum transfer $\hbar q$ is $q r_{N} \lesssim 1$ and that of the energy transfer $\hbar \omega$ may be given by $\sim \hbar q v_{T} \sim$ $\hbar q(T / m A)^{1 / 2}$, where $v_{T}$ is the thermal velocity of heavy nuclei, $T$ is the temperature, and $m$ is the mass of nucleons. Therefore the energy transfer relative to the thermal energy is $\delta \equiv \hbar \omega / T \lesssim \hbar r_{N}^{-1}(1 / m A T)^{1 / 2} \sim 0.6 A^{-1 / 2}$ for $r_{N}=10 \mathrm{fm}$ and $T=1 \mathrm{MeV}$. Provided $\delta$ is much smaller than unity, the assumption of elastic scattering is valid and effects of degeneracy of neutrinos can be also neglected [40]. However, at higher densities, where nucleons are strongly degenerate and the Fermi velocity of nucleons $v_{F} \sim c$, a typical energy transfer is $\sim \hbar q v_{F}$ and $\delta$ is no longer small for the above values of $q$ and $T$. Thus, in this case, the elastic assumption is not accurate [41, 42] and one should also take account of effects of degeneracy of neutrinos.

[14] G. Watanabe, K. Iida, and K. Sato, Nucl. Phys. A687, 512 (2001).

[15] G. Watanabe, K. Iida, and K. Sato, Nucl. Phys. A726, 357 (2003).

[16] J. M. Lattimer and F. D. Swesty, Nucl. Phys. A535, 331
(1992).

[17] K. Oyamatsu, Nucl. Phys. A561, 431 (1993).

[18] G. Baym, H. A. Bethe, and C. J. Pethick, Nucl. Phys. A175, 225 (1971).

[19] Because here we employ a liquid drop model in which the density profile of nuclei is approximated by a rectangular form, the resulting $\left\langle\bar{S}\left(E_{\nu}\right)\right\rangle$ is reliable only if the wavelength of neutrinos $\lambda_{\nu}$ is comparable to or larger than the typical size of the rectangular profile, $\lambda_{\nu} \gtrsim r_{N}\left(r_{N}\right.$ is the half width of nuclei). For, e.g., $r_{N}=10 \mathrm{fm}$, this condition reads $E_{\nu} \lesssim 120 \mathrm{MeV}$.

[20] C. P. Lorenz, D. G. Ravenhall, and C. J. Pethick, Phys. Rev. Lett. 70, 379 (1993).

[21] T. Maruyama, T. Tatsumi, D. N. Voskresensky, T. Tanigawa, and S. Chiba, Phys. Rev. C 72, 015802 (2005).

[22] We have also calculated $\left\langle\bar{S}\left(E_{\nu}\right)\right\rangle$ for another macroscopic model by Shen et al. [36]. The following discussion also holds for this model.

[23] A. D. Kaminker, C. J. Pethick, A. Y. Potekhin, V. Thorsson, and D. G. Yakovlev, Astron. Astrophys. 343, 1009 (1999).

[24] T. Maruyama, K. Niita, K. Oyamatsu, T. Maruyama, S. Chiba, and A. Iwamoto, Phys. Rev. C 57, 655 (1998).

[25] S. Chikazumi, T. Maruyama, S. Chiba, K. Niita, and A. Iwamoto, Phys. Rev. C 63, 024602 (2001).

[26] T. Kido, T. Maruyama, K. Niita, and S. Chiba, Nucl. Phys. A663, 877c (2000).

[27] We remark that the expression for the Hamiltonian given in Ref. 25] is erroneous. The quantity $L$ in the density dependent term [the second term in Eq. (2)] of that paper should be replaced by $\tilde{L}$ defined in the same way as in Ref. 24] (private communication with T. Maruyama).

[28] G. Watanabe, K. Sato, K. Yasuoka, and T. Ebisuzaki, Phys. Rev. C 66, 012801(R) (2002).

[29] G. Watanabe, K. Sato, K. Yasuoka, and T. Ebisuzaki, Phys. Rev. C 68, 035806 (2003).

[30] H. Sonoda et al., in preparation.

[31] We use simulation results of model 1 with 16384 nucleons and those of model 2 with 2048 nucleons. We have confirmed that the difference between $S(q)$ for simulations with 16384 and 2048 nucleons is small for model 1. However, we should mention that finite boundary effects of the simulation box might remain even for simulations with 16384 nucleons in the case of slab-like nuclei; the number of nuclei in the simulation box is around five in 
this case.

[32] W. H. Press, S. A. Teukolsky, W. T. Vettering, and B. P. Flannery, Numerical Recipes (Cambridge University Press, 1986).

[33] S. E. Woosley, A. Heger, and T. A. Weaver, Rev. Mod. Phys. 74, 1015 (2002).

[34] T. Takiwaki, K. Kotake, S. Nagataki, and K. Sato, Astrophys. J. 616, 1086 (2004).

[35] A. Heger, N. Langer, and S. E. Woosley, Astrophys. J. 544, 1016 (2000).

[36] H. Shen, H. Toki, K. Oyamatsu, and K. Sumiyoshi, Nucl. Phys. A637, 435 (1998).

[37] K. Kotake, S. Yamada, and K. Sato, Astrophys. J. 595, 304 (2003).
[38] C. J. Pethick and D. G. Ravenhall, Annu. Rev. Nucl. Part. Sci. 45, 429 (1995).

[39] Rapid rotation in the core drives the bounce by the strong centrifugal force, not by the nuclear repulsive force. In this case the central density just before the bounce is too small for the pasta phases to appear.

[40] D. Q. Lamb and C. J. Pethick, Astrophys. J. 209, L77 (1976).

[41] S. Reddy, M. Prakash, and J. M. Lattimer, Phys. Rev. D 58, 013009 (1998).

[42] A. Burrows and R. F. Sawyer, Phys. Rev. C 58, 554 (1998). 